\title{
Auf Seiten der Macht, des Geldes oder der Wahrheit? Eine Antwort an meine Kritiker
}

\section{Walter Herzog}

\author{
"Durch Wissenschaft wird nicht Sicherheit, \\ sondern ... Unsicherheit gesteigert.» \\ Niklas Luhmann (1992)
}

Ich bin der Redaktion der Schweizerischen Zeitschrift für Bildungswissenschaften dankbar, dass sie meinen Artikel einer Reihe von Kolleginnen und Kollegen zur Diskussion angeboten hat. Dadurch konnte ein wesentliches Anliegen, das mich zum Schreiben des Textes motiviert hat, nämlich eine Diskussion über ein praktisch undiskutiertes Thema anzuregen, bereits erfüllt werden. Die Gelegenheit, auf die elf Stellungnahmen zu antworten, nehme ich gerne wahr, denn sie ermöglicht mir, ein paar Punkte zu klären und Missverständnisse auszuräumen. Ich gehe auf die einzelnen Beiträge in der Reihenfolge ein, wie sie im Vorausgehenden abgedruckt sind. Kritiken, die mehrfach vorgebracht werden, diskutiere ich in der Regel nur an einer Stelle. Wo nicht anders angegeben, stammen die Zitate aus der jeweils diskutierten Stellungnahme.

(1) Roland Reichenbach stimmt meinen Ausführungen weitgehend - er sagt sogar «unumwunden» - zu. Verdienstvoll ist sein Versuch, die von mir monierte Theorielosigkeit der aktuellen Schulreform einer Erklärung zuzuführen. Deutlich wird dabei, dass das Ansinnen einer intensivierten Steuerung des Bildungssystems auf einer ungenügenden Theorie der Schule beruht, deren funktionale Vielfalt und anarchische Züge systematisch missachtet werden. Eigentlich könnten dies die Akteure der laufenden Reform durchaus wissen, denn es liegen genügend Erkenntnisse seitens der Bildungsforschung vor, die zeigen, dass Schule weder eine Mechanisierung ihrer Prozesse zulässt noch in Kategorien der industriellen Fertigung sinnvoll begriffen werden kann.

(2) Auch Daniel Bain pflichtet meiner Analyse im Wesentlichen bei, auch wenn sie ihm (wie einigen anderen auch) etwas polemisch erscheint. Bain greift ausführlich auf offizielle Dokumente zurück und beklagt das wenig transparente Vorgehen der EDK beim HarmoS-Projekt. Die Intransparenz erschwert die Kritik, ist aber ein weiteres Indiz für die Indienstnahme der Wissenschaft durch Po- 
litik und Verwaltung, denn ein offener Diskurs soll erkennbar nicht stattfinden. Im Übrigen betont auch Bain die simplizistische Sicht des Bildungssystems und seines Funktionierens sowie der Möglichkeiten seiner Reform, die dem HarmoS-Projekt zugrunde liegt. Von besonderem Gewicht sind die skeptischen Worte Bains zum diagnostischen Wert der in Entwicklung befindlichen Messinstrumente für die Bildungsstandards, kommen sie doch aus dem Munde eines Didaktikers. Bisher hatte man den Eindruck, die Fachdidaktiker hierzulande würden HarmoS als ein genuin unterrichtspraktisches Projekt wahrnehmen, was es aber offensichtlich nicht ist.

(3) Danièle Périsset Bagnoud versteht meine Ausführungen als Einladung zu einem Diskurs, dessen Fehlen im Vorfeld der Abstimmung über die neuen Bildungsartikel in der Schweizerischen Bundesverfassung sie mit einer gewissen Verwunderung vermerkt. Der Hauptakzent ihrer Stellungnahme liegt auf einer Reihe von offenen Fragen zu den Bildungsstandards sowie zu den Auswirkungen der laufenden Reformen auf den Lehrerberuf. Obwohl ich mich selber nur knapp zu letzterem Punkt äussern konnte, sehe ich bei Périsset Bagnoud eine weitgehende Übereinstimmung mit meinen eigenen warnenden Worten. Angesprochen sind die Pädagogischen Hochschulen, die sich bisher allerdings kaum zu den absehbaren Folgen des HarmoS-Projekts für den Lehrerberuf geäussert haben. Zu Recht betont Périsset Bagnoud, dass auch in Bezug auf den Lehrerberuf ohne theoretische Absicherung argumentiert wird. Die Begriffe «Profession» und "Professionalisierung» scheint jedermann nach Belieben mit Inhalt zu füllen. Kein Wunder, dass eine ernsthafte Diskussion nicht zustande kommt. Was die EDK meint, wenn sie in ihrem Tätigkeitsprogramm auch die «Stärkung der Professionalität der Lehrerinnen und Lehrer» (EDK 2006, S. 9) ausweist, bleibt gerade angesichts des HarmoS-Projekts völlig offen.

(4) Wenig Positives kann Bernard Schneuwly meinen Ausführungen abgewinnen. Allerdings hat er für seine Replik eine Flughöhe gewählt, aus der nur mehr grobe Konturen zu erkennen sind. Mag sein, dass meiner Argumentation ein Hang zum Dichotomischen innewohnt, doch kann ein rhetorisches Stilmittel schwerlich als Hinweis auf eine theoretische Position genommen werden. Da dies von Schneuwly verkannt wird, versteht er mich in wesentlichen Punkten falsch.

Das gilt schon für die «école artisanale romantique», der ich anhängen soll. Nur weil ich in meiner Herleitung des Standardbegriffs die industrielle Fertigung gegen die handwerkliche Produktionsweise ausspiele, kann man mich doch nicht zum Schulromantiker machen. Schneuwly entnimmt meinen Ausführungen das Bild eines charismatischen Pädagogen, «qui enseigne dans un lieu protégé, loin de toute pression sociale, de toute concurrence et sélection, selon les besoins des enfants, suivant leur développement spontané, inventant outils, méthodes et techniques». Ist dies das Ergebnis einer sorgfältigen Lektüre meines Textes? Wohl kaum. Wenn mich Schneuwly zudem mit Ferrière in eine Ecke stellt, dann entbehrt dies nicht einer gewissen Komik. Für eine solche Karikatur 
reichen auch meine Ausführungen zur Schule als «Lebenseinheit» nicht hin, denn dort referiere ich lediglich ein paar Punkte aus einer Liste von Fend, die auf das Zwecklose der Schule verweisen, ohne dass ich sage, die Schule solle nur dies sein! Nirgends schliesse ich aus, dass die Schule auch ein Ort des Lernens und der Leistung sein soll. Schneuwly postuliert einfach, dass ich, weil ich die ThorndikeTradition kritisiere, auf der anderen Seite stehen muss, die er mit dem Namen von Dewey belegt. Die Unterscheidung ist etwa so scharfsinnig, wie wenn man in Bezug auf den deutschen Sprachraum sagen wollte, es bestehe neben der Pädagogik auch eine Reformpädagogik. ${ }^{1}$

Wie uninspiriert Schneuwlys Lektüre meines Textes ist, zeigt auch die Einweisung meiner Person ins Lager der Popperianer. Popper zitiere ich ausschliesslich, um das Argument, wonach Wissenschaft weder auf reinen Fakten noch auf reinen Gedanken beruht, zu bekräftigen. Diese Position kann man ohne weiteres auch mit anderen Autoren belegen, wie Jean-Luc Patry zeigt, der auf Dewey und das Prinzip der "warranted assertibility» verweist. Eine weitere Möglichkeit wäre Luhmann (1992): "Weder kann man von sich selbst evidenzierenden Wahrnehmungen, noch von intuitiv sicheren Axiomen ausgehen» (S. 462). Gerade Popper wäre kein Beleg für die Ansicht, wonach Wissenschaft auf diskursiver Argumentation beruht, hat er doch bis zuletzt an seiner Drei-Welten-Lehre festgehalten und als Basis der wissenschaftlichen Beweisführung nur die zweiwertige, deduktive Logik anerkannt. Selbst das Killerargument des Relativismus und das Schreckgespenst des Elfenbeinturms sind Schneuwly nicht zu schade, um meine Position ins Lächerliche zu ziehen. Sage ich denn irgendwo, die Messungen, die man im Rahmen von HarmoS durchführt, seien ohne Realitätsbezug? Die Frage ist doch nicht, ob wissenschaftliche Erkenntnisse einen Realitätsbezug haben oder nicht, sondern ob sie wahr oder falsch sind.

Aus Platzgründen kann ich auf weitere Schnitzer in Schneuwlys Replik nicht eingehen. Nur eines sei noch vermerkt: Schneuwlys eigener Hang zur Romantik. Überall objektive Widersprüche («contradictions inhérentes») zu sehen und den Widerspruch zum Motor der Veränderung («moteur du développement») zu stilisieren, erinnert mich lebhaft an meine Studienzeit, als solche «Grossen Erzählungen» (Lyotard) noch für glaubhaft gehalten wurden.

(5) Ganz anders als von Schneuwlys Verriss bin ich vom Beitrag von Christian Maroy und Vincent Dupriez sehr angetan. Obwohl auch sie in meinem Text regressive Fantasien vermuten - meine Kritik soll «sur une conception «humaniste et (artisanale) de l'éducation, de ses visées et modes de fonctionnement» beruhen -, stellen sie meine Position im Wesentlichen richtig dar. Ähnlich wie Reichenbach ergänzen sie meine deskriptiv gehaltenen Ausführungen um eine theoretische Skizze, die erklären kann, weshalb das Bildungswesen unter den Einfluss ökonomischer Denkweisen geraten ist. Die soziologische Analyse, in die sie meine Kritik einbetten, postuliert eine Abfolge von einer bürokratischen zu einer post-bürokratischen Organisation von Schule, die mit einem Verlust an politischer Legitimation einhergeht. Die Schwächung des institutionellen Charakters der 
Schule führt zu einer eindimensionalen Ausrichtung von Steuerungsentscheiden an Kriterien der Zweckrationalität. Ausschlaggebend ist nur mehr «ce qui marche». Die Schule wird überrollt von einer Performanzkultur (Radtke, 2002), die ohne Zieldiskurse auszukommen scheint. ${ }^{2}$

Maroy und Dupriez betonen den erhöhten Machtanspruch, der mit dem post-bürokratischen Modell verbunden ist, ein Machtanspruch, der sich negativ auf die Professionalisierung des Lehrerberufs auswirkt. Die beiden Kollegen verweisen zudem auf den Apparat an Experten und Technokraten, der die Reform begleitet und umsetzt. Davon kann man inzwischen auch in der Schweiz Kenntnis nehmen. NPM zum Trotz werden die Bildungsverwaltungen aufgebläht, und es scheint, dass uns hier die eigentlichen Agenten der Harmonisierung des schweizerischen Schulsystems vor Augen stehen. Für die Disziplin Erziehungswissenschaft ist dies nicht ohne Ambivalenz. Denn ein aufgeblasener administrativer Apparat schafft auch Arbeitsplätze für Abgängerinnen und Abgänger der erziehungswissenschaftlichen Studiengänge. Unweigerlich geraten die frisch gebackenen Kolleginnen und Kollegen aber in ein Dilemma: Sollen sie sich nun der Wissenschaft (Wahrheit) oder der Politik (Macht) verpflichten?

(6) Gianni Ghisla empfindet meine Kritik an der Ökonomisierung des Bildungswesens als zu aufgeregt. Wobei ich den Eindruck habe, dass unsere Positionen so verschieden gar nicht sind. Was Ghisla zu den Bildungsstandards ausführt, stimmt mit meinen eigenen Ansichten überein. Auch seiner Auseinandersetzung mit dem Kompetenzbegriff kann ich problemlos zustimmen. Unklar ist mir jedoch bis zuletzt geblieben, wie sich Ghisla zu der von ihm so genannten «ökonomischen Finalisierung von Bildung, Forschung und Innovation» stellt. Es beginnt schon mit den Zitaten von Gary Becker. Stimmt er ihnen zu? Oder ist er einfach fasziniert von ihnen? Wer alles zur Ökonomie erklärt, kann gerade so gut auch alles zur Idee, zum Schein oder zum Widerspruch erklären. Erkenntnis ist doch nur möglich, wo nicht alles einerlei ist, sondern wo Differenzen und Unterschiede bestehen. Also muss auch die Ökonomie ihre Grenzen haben, sonst würde sie schlicht nichts erklären. Die Auseinandersetzung mit der Ökonomie, die Ghisla fordert, müsste dann aber genau dies sein: ein Versuch, die Grenzen des ökonomischen Denkens (auch) für die Bildung zu bestimmen.

Vielleicht sind unsere Positionen aber auch diesbezüglich nicht wirklich verschieden, denn später appelliert Ghisla an die «Fähigkeit der Bildungspolitik, Bildung nicht zur Ware degradieren zu lassen». Unmittelbar danach heisst es aber wieder, Appelle an die Bildungspolitik "wider die Instrumentalisierung" (der Erziehungswissenschaft) würden «realistischerweise ... nicht viel (bringen)». Sind wir der Wirtschaft und ihrem Medium Geld nun ausgeliefert oder nicht? Da mir Ghisla vorwirft, die Erziehungswissenschaft als «Opfer rücksichtsloser Politik» darzustellen, scheint er anzunehmen, dass sich durchaus etwas machen lässt. Nur kommt ihm dann seine Schelte der Erziehungswissenschaft in die Quere, die seiner Ansicht nach zuerst vor der eigenen Türe kehren müsste. Mag 
sein, dass mit der Erziehungswissenschaft nicht Alles zum Besten steht und es sinnvoll wäre, wenn sie (wieder) «einmal radikal über die Bücher» ginge. Doch scheint mir dies ein anderes Thema zu sein als dasjenige, das ich in meinem Artikel anspreche. Und es wäre fatal, durch den Hinweis auf die Schwächen der Erziehungswissenschaft davon abzulenken, dass sie einer Attacke von Seiten einer ökonomisch inspirierten Politik ausgesetzt ist.

(7) Etwas geärgert habe ich mich über den Beitrag von Esther Berner. Die Kollegin unterstellt mir Dinge, die ich in keiner Weise vertrete und meinem Artikel auch nicht entnommen werden können. Wo sage ich denn, Kompetenzen hätten «nichts» mit Wissen zu tun? Wo fordere ich eine «neue Bildungstheorie, die ... Empirie und Theorie trennt»? Wo verlange ich eine nochmalige Vergewisserung über die Struktur der pädagogischen Wirklichkeit? Woraus lässt sich ableiten, ich sei ein Gegner, gar ein bekennender Gegner von Bildungsstandards? Weshalb soll es ein Widerspruch sein, wenn ich die mit Standards einhergehende Normierung kritisiere, dies aber auch in Bezug auf den formalen Charakter von Kompetenzstufen tue?

Auch Berner muss ich darauf hinweisen, dass meine Gegenüberstellung von industrieller Fertigung und handwerklicher Herstellung im Kontext der Erläuterung des Standardbegriffs steht. Dass ich «ein Verständnis von Erziehungs- und Unterrichtsprozessen [bevorzuge], das sich mit demjenigen der handwerklichen Produktion` vergleichen lässt», ist eine schlichte Behauptung. Gleiches gilt für die Aussage, ich würde die Berücksichtigung lokaler Bedingungen fordern. Allerdings bin ich durchaus der Meinung, dass Schule und Unterricht faktisch immer unter Bedingungen stattfinden, die eine abstrakte Gleichbehandlung und Gleichbeurteilung sowohl der Lehrkräfte wie der Schülerinnen und Schüler nicht zulassen. Die lokalen und situativen Bedingungen pädagogischen Handelns wegdiskutieren zu wollen, gar mit dem Argument der Gerechtigkeit, halte ich für ziemlich schief. ${ }^{3}$ Die Situativität von Schule und Unterricht hat zudem eine forschungsmethodische Seite. Neben der unabsehbaren Zahl von Interaktionen, ist es vor allem die Kontextualität pädagogischer Abläufe, die aus der Erziehungswissenschaft eine der anspruchsvollsten Disziplinen macht (Berliner, 2002).

Weiter moniert Berner, ich würde nicht erwähnen, dass es sich bei den schweizerischen Bildungsstandards um «Mindeststandards» handelt. Selbst wenn dies stimmen würde - tatsächlich stimmt es nicht, denn ich verweise gleich zu Beginn des ersten Abschnitts meines Artikels auf die "Mindest- bzw. Basisstandards», die dem EDK-Projekt zugrunde liegen -, wäre doch klar, dass Mindeststandards nicht an sich festgelegt werden können. Wie Ghisla darlegt, liegen Standards auf einem "Qualitätskontinuum». Es sind "cutscores», die eine Skala voraussetzen, auf der sie als Markierung eingetragen werden (Mehrens \& Cize, 2001, S. 477) - als Mindeststandard, als Maximalstandard oder irgendwo dazwischen (zum Beispiel als Regelstandard).

Auch die Kritik, ich würde in meiner Darstellung des bildungspolitischen Steuerungsmodells die «Feedback-Schleife» unterschlagen, ist nicht nachzuvoll- 
ziehen. Wer die Texte zum Bildungsmonitoring liest, kann doch schwerlich übersehen, wie Politik und Wissenschaft miteinander verknüpft werden sollen. Hier liegt sogar mein hauptsächlicher Kritikpunkt! Wie kann ein kybernetisches Modell mit negativer Rückkopplung, das für die Regulation von Kühlsystemen beste Dienste leistet, für die Analyse eines komplexen Gefüges, wie es ein Bildungssystem darstellt, tauglich sein? Das Modell ist einfach zu simpel, als dass es leisten könnte, was man ihm abverlangt.

Ich verzichte darauf, die weiteren Missverständnisse in Berners Stellungnahme auszuräumen. Wer auszieht, die «argumentative Qualität» eines Textes zu bemängeln und dessen Autor gleich mehrerer "Irrtümer» zu bezichtigen, müsste auf die Qualitätssicherung seiner eigenen Darlegungen etwas mehr achten, als dies Berner getan hat.

(8) Der Text von Marcel Crahay reiht sich in die kleine Gruppe von Stellungnahmen ein, die meine deskriptiv bleibende Analyse um Erklärungsansätze erweitern. Ich stimme Crahay bedingungslos zu, dass eine Kritik von HarmoS in abstracto nicht genügen kann und in einen gesellschaftlichen und historischen Kontext eingebettet werden muss. Wie Reichenbach sowie Maroy und Dupriez liefert Crahay diese Einbettung auf brillante Weise nach, so dass eine offensichtliche Schwäche meines Textes nun behoben ist.

Crahay bestreitet, dass die aktuellen Reformen theorielos seien, wie ich in meinem Artikel behaupte. Die Theorien seien vorhanden und kämen aus der Ökonomie. Damit bin ich allerdings einverstanden. Die Kritik der ökonomischen Provenienz der Reformansätze bildet sogar ein wesentliches Element meiner Argumentation. Offensichtlich habe ich mich missverständlich ausgedrückt. Was ich meine, ist nicht, dass theoretische Konzepte nicht vorliegen, sondern dass es sich um (fremde) Theorien handelt, deren Relevanz für die Analyse pädagogischer Sachverhalte nicht nachgewiesen ist. Die blosse Übertragung einer ökonomischen (oder irgend einer anderen) Theorie auf den Bereich der Bildung ist als wissenschaftliche Grundlage für eine dermassen weitgehende Reform unzureichend. Mein Argument ist nicht, dass man Bildung und Erziehung nicht auch unter ökonomischen Kategorien beurteilen kann - das kann und soll man durchaus -, sondern dass ökonomische Denkmodelle auf das Bildungswesen angewandt werden, ohne dass die Angemessenheit einer solchen Anwendung empirisch überprüft worden wäre.

(9) Als sehr differenziert empfinde ich die Stellungnahme von Jean-Luc Patry. Obwohl ihm der polemische Stil meines Textes nicht zu behagen scheint, sind seine Ausführungen äusserst hilfreich. Er bringt ein Moment ins Spiel, das sonst - mit Ausnahme der Bemerkungen von Bain zur diagnostischen Validität - weitgehend ausgespart bleibt: die methodischen und messtheoretischen Probleme von HarmoS und ähnlichen Reformprojekten. Patry macht nachvollziehbar, weshalb Bildungsstandards nicht ohne weiteres für alle schulischen Fächer und schon gar nicht für die erzieherischen Ziele der Schule entwickelt werden können. Damit stützt er ein wesentliches Argument meines Textes, dass nämlich Bil- 
dungsstandards und Bildungsmonitoring die Schule in ein Licht rücken, das unzulässig vieles ausblendet, was von ihr nicht weggedacht werden kann.

Patrys Aussagen zu «Input und Output» sind treffend. Tatsächlich umschreiben Lehrpläne (qua Input) die Ergebnisse von Bildungsprozessen. Sprachlich stehen sie oft im Indikativ («Der Schüler beherrscht die Grundrechenoperationen.» oder: «Der Schüler kann ein kurzes Gedicht frei vortragen.»). Damit nehmen sie gleichsam vorweg, was allererst erreicht werden soll. Umgekehrt ist die "Outputsteuerung» keine Steuerung des Outputs, sondern weiterhin eine Steuerung des Inputs, die sich allerdings an Informationen über den Output orientiert. Wenn Patry meint, die Trennung von Input und Output könne im Unterricht «nicht ganz durchgezogen werden», dann fliesst Wasser auf meine Mühle, da ich ja bezweifle, dass simple Input-Output-Modelle für die Analyse pädagogischer Prozesse tauglich sind. Zudem verweist Patry auf ein ethisches Moment, das ich ebenfalls teile und das seit Schleiermacher (1826/2000) zum Grundbestand pädagogischen Denkens gehört: Niemand hat das Recht, die Gegenwart eines Kindes seiner Zukunft zu opfern. Auch deshalb gehört die Zwecklosigkeit zwingend mit zur Idee von Schule.

Nicht einverstanden bin ich mit Patrys Lob des TOTE-Modells, nicht weil es ein «Ladenhüter» wäre, sondern weil es auf die Situation nicht anwendbar ist, von der mein Text handelt. Das TOTE-Modell ist ein psychologisches Modell, das als Einheit eine klare Referenz hat: das handelnde Individuum. Die Unterrichtssituation stellt aber eine andere Einheit dar, nämlich eine soziale, die aus mehreren Individuen besteht. Wie das kybernetische Kühlschrankmodell genügt das TOTE-Modell nicht, um die Komplexität einer Unterrichtssituation zu erfassen. Die einfache Logik des "plan, do, monitor and review» (Gleeson \& Gunter, 2001, S. 145) lässt sich befolgen, wo auch die Verhältnisse einfach sind, aber nicht, wo sie komplex sind. Insofern ist das TOTE-Modell ein gutes Beispiel für die Suggestibilität von linearen Prozessschemata, die für sich genommen zwar plausibel scheinen, dem erziehungswissenschaftlichen Gegenstandes aber nicht gerecht werden.

(10) Maurice Tardif wirft mir Verteufelung des HarmoS-Projekts vor, eine etwas scharfe Wortwahl, die nur leicht abgemildert wird, wenn er zudem feststellt, ich sei mit der Axt ans Werk gegangen. Auch Tardif moniert ein dichotomisches Denken («système bipolaire») in meinem Artikel, wobei er nicht zwei Konzeptionen von Pädagogik vor Augen hat, sondern einen Dualismus von Erziehungswissenschaft und Ökonomie. Wie Crahay erinnert Tardif an die engen Beziehungen zwischen der Industrialisierung der Gesellschaft und der Einrichtung von Schulen im 19. Jahrhundert. Ich bin der Letzte, der bezweifeln wollte, dass zwischen Bildung und Wirtschaft enge Beziehungen bestehen. Allerdings halte ich ebenso sehr für eine systemische Betrachtungsweise ihres Verhältnisses. In einer funktional differenzierten Gesellschaft operieren die sozialen Teilsysteme nach eigener Logik, was sie relativ autonom macht (Luhmann, 1997). Kein System kann mittels seines Codes an den Code eines anderen Systems anschliessen, 
womit der Wirtschaft und der Politik nicht nur hinsichtlich der Wissenschaft, sondern auch bezüglich der Bildung Grenzen gesetzt sind.

Insofern habe ich etwas Mühe, wenn Tardif die Erziehung und die Schule tel quel als ökonomische Realitäten darstellt («des réalités profondément économiques»). Zweifellos verursacht das Bildungssystem Kosten, und hier liegt ohne Zweifel ein wesentlicher Grund für die aktuellen Schulreformen, was von verschiedener Seite denn auch vermerkt wird. Diese Einsicht darf aber nicht dazu führen, dass das Bildungswesen dem Denken in ökonomischen Kategorien unterworfen wird. Ich meine ja nicht, die Schulen seien unschuldige Jungfrauen, deren fleischliche Gelüste erst durch die Wirtschaft geweckt würden (wie die Metaphorik von Tardif suggeriert). Aber auch die Universitäten kosten viel, und trotzdem wird in keiner Stellungnahme die Meinung vertreten, die Wissenschaft hätte sich dem Code der Ökonomie zu beugen. Die Freiheit der Forschung ist Voraussetzung dafür, dass sich die Wahrheit Gehör verschaffen kann. Genau deshalb darf nicht geschehen, dass die Erziehungswissenschaft ökonomischen Zwecksetzungen unterworfen wird. Das hat nichts mit dem Anspruch auf «reine» Wissenschaft zu tun, wie mir Tardif zu unterstellen scheint («vision épurée et noble»), sondern mit der schlichten Tatsache, dass die Wahrheit - als Code der Wissenschaft (Luhmann, 1992) - an Bedingungen gebunden ist, die weder im politischen noch im ökonomischen System gegeben sind.

Wie Tardif völlig zu Recht darlegt, sind die Bildungswissenschaften («sciences de l'éducation») in enger Verknüpfung mit staatlichen Interessen entstanden. Dies gilt nicht nur für Nordamerika, sondern auch für Europa. Insofern behaupte ich auch nicht, wir hätten es mit einem neuen Phänomen zu tun. Doch gehe ich sehr wohl davon aus, dass genau hier ein Problem der Legitimität der Erziehungswissenschaft als akademischer Disziplin liegt. Anders als Tardif, der hier offenbar keinen Zusammenhang sieht, meine ich, dass sich die monierten Schwächen der erziehungswissenschaftlichen Forschung (auch) der Tatsache verdanken, dass die Disziplin Ansprüchen ausgesetzt ist, die nicht wissenschaftlicher Natur sind. Tardif dreht meine Argumentation um, wenn er meint, die Gründe für die epistemische Inkompetenz der Erziehungswissenschaft würden in ihr selber liegen, während ich meine, dass sie zum grossen Teil extern bedingt sind. In diesem Punkt hebe ich mich auch von all jenen Kolleginnen und Kollegen ab, die versuchen, aus der Not eine Tugend zu machen, indem sie der Erziehungswissenschaft einen besonderen Charakter als Wissenschaft zuweisen: als Handlungswissenschaft, als praktische Wissenschaft, als Wissenschaft von der Praxis für die Praxis, als Berufswissenschaft für Lehrer etc. Auf diesem Dampfer befinde ich mich eindeutig nicht. Aber deshalb von einer "Jungakademiker-Illusion» (Criblez) zu sprechen, halte ich für verfehlt.

(11) Auch die Ausführungen von Lucien Criblez reihen sich in jene Gruppe von Stellungnahmen ein, die meiner Argumentation etwas mehr Tiefenschärfe geben wollen, indem sie nach Erklärungen dessen suchen, was ich kritisiere. Etwas eigenartig mutet allerdings an, wenn Criblez von einer «anderen Lesart» der 
Ereignisse spricht, wirft er mir doch gleichzeitig Ahistorizität vor. Criblez präsentiert meines Erachtens nicht eine «andere Lesart», sondern untermauert meine Ausführungen mit historischem Material. Dabei kann ich seiner Argumentation, die besonders die politische Situation in der Schweiz fokussiert, durchaus folgen. Ich habe auch nicht behauptet, die Bildungspolitik wolle die Erziehungswissenschaft unmotiviert für ihre Interessen in Dienst nehmen. Erstaunt bin ich jedoch über eine gewisse Naivität, die Criblez Darlegungen durchzieht. Genügt es für eine historische Analyse, das Selbstverständnis der Akteure zu rekonstruieren und sich auf offizielle Dokumente zu stützen? Anders als Reichenbach, Maroy und Dupriez sowie Crahay bemüht sich Criblez nicht um eine theoretische Erweiterung meiner Analyse, sondern verbleibt im Narrativen, was seinen Ausführungen einen leicht affirmativen Charakter gibt.

Um ein Beispiel zu geben, scheint es mir etwas fragwürdig zu sein, staatliche Interventionen damit zu sanktionieren, dass man ihnen zuschreibt, sie hätten immer eine Wirkung, wenn nicht die erwartete, dann eine unerwartete. Mir wird jeweils unwohl, wenn ich in Bezug auf PISA oder andere Schulleistungsstudien höre, obwohl sie nichts bringen, würden sie wenigstens eine Diskussion auslösen. Solche zynisch wirkenden Voten geben Reichenbach Recht, der in Schulreformen eine Art Selbstläufer sieht, der immer wieder neu in Bewegung kommt, weil eine «rationale Politik〉 gar nicht möglich ist!

Insofern stört mich, dass Criblez den Steuerungsbegriff undiskutiert lässt. Wenn Politiker ein Wort verwenden, dann mag man hinnehmen, dass es sich oft um eine façon de parler handelt. In der Wissenschaft sollte es aber anders sein. Wie viel «Steuerung» ist denn in einem Politikbereich möglich, der von Widersprüchen durchzogen ist (Schneuwly), multiplen Funktionen genügen muss (Reichenbach), dessen Wirkmechanismen bestenfalls ansatzweise bekannt sind und der in einem unauflöslichen Konflikt mit der Wissenschaft steht (Criblez)? Selbst wenn die Steuerung lediglich auf der Makro- und Mesoebene ansetzen sollte, bleibt völlig offen, wie und was denn gesteuert werden kann. Criblez verkennt auch, dass ich nicht behaupte, die aktuellen Schulreformen würden auf eine Steuerung der Unterrichtsebene zielen. Mein Punkt ist vielmehr, dass die technologische Mentalität, die sich in Bildungspolitik und -verwaltung breit macht, in die Öffentlichkeit diffundieren und der Schule sowie dem Lehrerberuf zum Schaden gereichen könnte. ${ }^{4}$

Criblez stellt das Denken in Kategorien der Steuerung selber in Frage, wenn er das Verhältnis von Wissenschaft und Politik systemisch deutet. Wie aber kann er beides unter einen Hut bringen: die Ambition der Steuerung und die Einsicht, dass Steuerung nicht möglich ist? Und was soll die Rede von «bildungspolitischer Steuerung» und «Steuerungswissen», das der Bildungsforschung von Seiten der Politik - gemäss Criblez offenbar zu Recht - abverlangt wird? Wie bei Tardif mündet die Argumentation Criblez' in ein quietistisches Schulterzucken: Meine «Besorgnis um eine autonome Erziehungswissenschaft» sei zwar «ehrenhaft», aber da die Verhältnisse nun mal sind wie sie sind, nehmen wir sie halt hin 
- am besten gleich mit der von Ghisla empfohlenen Gelassenheit.

Criblez unterbietet meine Kritik, wenn er auf die Legitimität einer Politik hinweist, die Entscheidungen wissenschaftlich abstützt. Dagegen habe ich doch nichts! Mein Punkt ist nicht die Alimentierung politischer Entscheidungen mit wissenschaftlicher Erkenntnis, sondern die Indienstnahme der Wissenschaft durch eine Politik, die sich als rational ausgibt. Gegen dieses Ansinnen hilft der Aufruf an die Erziehungswissenschaft, «die eigene Disziplin [zu] stärken», wenig. Wie im Falle des Lehrerberufs kann man nicht autonom sein, wenn einem die nötige Autonomie nicht gewährt wird. Dabei rede ich nicht der "vollständigen Autonomie der Wissenschaft» das Wort. Der Vorwurf ist unfair und hat einzig zur Folge, dass dem Status Quo die Absolution erteilt wird. Fast kommt es mir vor, als zünde Criblez eine Nebelpetarde, damit sich die Konturen meiner Kritik im Dunst verwischen und schnell wieder zur Tagesordnung zurückgekehrt werden kann. Vielleicht liegt das in seinem Interesse, in meinem liegt es nicht.

Noch ein Wort zum "Nationendenken», das nicht nur Criblez, sondern auch Schneuwly und Patry als Kritik an den Bildungsstandards zurückweisen. Was ich meine, ist allerdings nicht die Rangierung von Nationen bzw. Kantonen, wie sie uns von PISA vorgemacht wird. Insofern ist die Bezugnahme auf PISA an besagter Stelle in meinem Text irreführend. Gemeint ist, dass die Reform der Schule ausgerechnet an den Grenzen der Nation innehält. Indem jedes Land seine eigenen «nationalen Bildungsstandards` entwickelt, rückt die Nation sehr wohl in den Vordergrund, während in anderen Politikbereichen die staatlichen Grenzen an Bedeutung verlieren oder aufgelöst werden (Marginson, 1999). Eine ökonomisch motivierte Politik benutzt das Bildungswesen, um der einheimischen Wirtschaft auf den globalen Märkten höhere Kompetitivität zu verschaffen. Ist dies kein Nationendenken?

Ein letzter Punkt betrifft die Frage der demokratischen Legitimität einer Politik, die sich auf (gesichertes Wissen` und «erwiesene Kausalitäten` beruft. Criblez spricht das Problem kurz an, um es allerdings gleich wieder fallen zu lassen. Auch in anderen Stellungnahmen wird es aufgegriffen, aber ebenfalls nur im Vorbeigehen (so z.B. bei Schneuwly in Bezug auf die demokratische Kontrolle der Entscheidungen der EDK). "Gesicherte Erkenntnisse» (auch Criblez verwendet diesen unsäglichen Ausdruck) liegen weit ab von demokratischen Entscheidungen, denn was ‘gesichert) und «erwiesen` ist, bedarf keiner Zustimmung durch eine Mehrheit. Insofern entzieht sich eine auf Evidenzen basierte Politik der öffentlichen Legitimation. Auch wenn in den neuen Artikeln der Bundesverfassung von einem «Bildungsraum Schweiz» (Art. 61a) die Rede ist und die Kantone sich zur «Harmonisierung des Schulwesens» (Art. 62) aufgerufen sehen, steht nirgends geschrieben, dass dies auf Kosten der politischen Öffentlichkeit und zu Lasten demokratischer Verfahren gehen soll. Allein schon der Versuch, die Erziehungswissenschaft im Rahmen einer «evidence-based policy» für bildungspolitische Zwecke zu instrumentalisieren, kann in einem Land wie der Schweiz mit seiner basisdemokratischen Tradition nicht hingenommen werden! 


\section{Anmerkungen}

1 Schon auf die USA bezogen ist die Dichotomie falsch, denn neben Thorndike und Dewey wäre auch Judd zu berücksichtigen (Hilgard, 1996; Lagemann, 2000).

2 Die Marginalisierung der Diskussion um Normen und Ziele der Schule wird unter dem Stichwort der «Kulturbereinigung» auch von Gianni Ghisla erwähnt und durch eine treffende Anekdote illustriert.

3 Für eine nach wie vor überzeugende Darstellung der Situativität pädagogischen Handelns vgl. Patry (1991), jetzt auch Patry (2000).

4 Vielleicht sollte man der Begrifflichkeit genau deshalb ihre Anschaulichkeit nehmen und statt von «Steuerung» von «Governance» sprechen (Kussau \& Brüsemeister, 2007).

\section{Literaturverzeichnis}

Berliner, D. C. (2002). Educational Research: The Hardest Science of All. Educational Researcher, 31 (8), 18-20.

EDK [Schweizerische Konferenz der kantonalen Erziehungsdirektoren] (2006). Interkantonale Vereinbarung über die Harmonisierung der obligatorischen Schule. HarmoS-Konkordat. Bericht zur Vernehmlassung. Bern: EDK.

Gleeson, D. \& Gunter, H. (2001). The Performing School and the Modernisation of Teachers. In D. Gleeson \& C. Husbands (Eds.), The Performing School. Managing Teaching and Learning in a Performance Culture (pp. 139-158). London: RoutledgeFalmer.

Hilgard, E. R. (1996). History of Educational Psychology. In D. C. Berliner \& R. C. Calfee (Eds.), Handbook of Educational Psychology (pp. 990-1004). New York: Macmillan.

Kussau, J. \& Brüsemeister, Th. (2007). Governance, Schule und Politik. Zwischen Antagonismus und Kooperation. Wiesbaden: Verlag für Sozialwissenschaften.

Lagemann, E. C. (2000). An Elusive Science. The Troubling History of Education Research. Chicago: University of Chicago Press.

Luhmann, N. (1992). Die Wissenschaft der Gesellschaft. Frankfurt a.M.: Suhrkamp.

Luhmann, N. (1997). Die Gesellschaft der Gesellschaft (2 Bde.). Frankfurt a.M.: Suhrkamp.

Marginson, S. (1999). After Globalization: Emerging Politics of Education. Journal of Education Policy, 14, 19-31.

Mehrens, W. A. \& Cizek, G .J. (2001). Standard Setting and the Public Good: Benefits Accrued and Anticipated. In G. J. Cizek (Eds.), Setting Performance Standards. Concepts, Methods, and Perspectives (pp. 477-485). Mahwah, N.J.: Lawrence Erlbaum.

Patry, J.-L. (1991). Transsituationale Konsistenz des Verhaltens und Handelns in der Erziehung. Bern: Lang.

Patry, J.-L. (2000). Kaktus und Salat. Zur Situationsspezifität in der Erziehung. In J.-L. Patry \& F. Riffert (Hrsg.), Situationsspezifität in pädagogischen Handlungsfeldern (S. 13-52). Innsbruck: Studienverlag.

Radtke, F.-O. (2002). Die Erziehungswissenschaft der OECD. Aussichten auf die neue Performanz-Kultur. In D. Nittel \& W. Seitter (Hrsg.), Die Bildung des Erwachsenen. Erziehungs- und sozialwissenschaftliche Zugänge (S. 277-304). Bielefeld: Bertelsmann.

Schleiermacher, F. (1826/2000). Texte zur Pädagogik. Kommentierte Studienausgabe Bd. 2. Frankfurt a.M.: Suhrkamp. 



\title{
Du côté du pouvoir, de l'argent ou de la vérité? Une réponse à mes discutants critiques
}

\section{Walter Herzog}

\author{
"La science n'accroît pas la sécurité, \\ mais... l'insécurité».
}

Niklas Luhmann (1992)

Je suis reconnaissant envers la rédaction de la Revue suisse des sciences de l'éducation d'avoir proposé mon article à un certain nombre de collègues pour discussion. Ainsi, l'une des principales préoccupations qui m'a motivé à écrire ce texte, actuellement ainsi satisfaite, a notamment été d'initier un débat sur un objet quasiment non discuté. C'est avec plaisir que je profite de l'occasion pour répondre à chacune des 11 prises de position, ce qui me permet aussi de clarifier quelques points et de dissiper certains malentendus. Je vais répondre à ces différentes contributions dans l'ordre dans lequel elles sont publiées dans le présent numéro spécial. Les critiques exprimées dans plusieurs textes ne font en principe l'objet que d'une seule réponse. Sauf indication contraire, les citations proviennent de la contribution discutée.

(1) Roland Reichenbach approuve largement mes positions - il dit même: «sans détours». Sa tentative d'expliquer le manque, que j'ai critiqué, de théorie de la réforme scolaire actuelle est louable. Cela démontre clairement que l'idée d'un pilotage intensifié du système éducatif repose sur une théorie incomplète de l'école dont la diversité fonctionnelle et les tendances anarchiques sont systématiquement ignorées. En fait, les acteurs de la réforme en cours pourraient le savoir, car il existe suffisamment de connaissances du côté de la recherche en éducation qui montrent que l'école ne peut se traduire par une mécanisation de ses processus et ne peut être appréhendée à l'aide de catégories identiques à celles de la fabrication industrielle.

(2) De même, sur les points principaux, Daniel Bain partage en substance mon analyse, même si elle lui semble (comme à d'autres également) quelque peu polémique. Bain reprend en détail des documents officiels et déplore la démarche peu transparente de la CDIP dans le projet HarmoS. Ce manque de transparence rend difficile la critique et ceci représente un indice de l'asservissement 
de la science par le politique et l'administration, aucun débat ouvert ne devant manifestement avoir lieu. Par ailleurs, Bain souligne également la vision simpliste du système éducatif, de son fonctionnement et des possibilités de le réformer, vision sur laquelle se base le projet HarmoS. Les propos sceptiques de Bain sur la valeur diagnostique des instruments de mesure pour les standards en éducation, actuellement en cours d'élaboration, ont un poids particulier puisqu'ils proviennent de la plume d'un didacticien. Jusqu'à présent, on avait l'impression que les didacticiens percevaient $\mathrm{HarmoS}$ comme un projet d'enseignement pratique, ce qu'il n'est visiblement pas.

(3) Danièle Périsset Bagnoud comprend mes explications comme invitation à un débat, dont elle note avec un étonnement certain l'absence à la veille du vote sur le nouvel article de la formation dans la Constitution fédérale suisse. L'accent principal de sa prise de position est mis sur une série de questions ouvertes, liées aux standards de la formation et aux effets des réformes en cours sur le métier d'enseignant. Bien que je me sois à peine prononcé moi-même sur ce dernier point, je vois chez Périsset Bagnoud une large concordance avec mes propres mises en garde. Elles s'adressent aux hautes écoles pédagogiques qui jusqu’à présent ne se sont guère prononcées sur les conséquences prévisibles du projet HarmoS pour la profession des enseignants. À juste titre, Périsset Bagnoud souligne que même en ce qui concerne le métier d'enseignant, l'argumentation est sans fondement théorique. Chacun semble donner à sa guise sa propre définition aux notions de "profession» et "professionnalisation". Pas étonnant qu'une discussion sérieuse n'ait pas lieu. Ce que la CDIP exige en mentionnant aussi «le renforcement de la professionnalité des enseignantes et des enseignants» dans son programme d'activité (CDIP, 2006, p. 9) reste totalement ouvert par rapport au projet HarmoS.

(4) Bernard Schneuwly ne retire que peu de points positifs de mes développements. À vrai dire, il a opté pour un niveau d'abstraction qui ne permet d'en voir que de vagues contours. Peut-être mon argumentation inclut-elle une tendance à la dichotomie, cependant un style rhétorique peut difficilement être compris comme l'indication d'une position théorique. Comme Schneuwly méconnaît cela, il me comprend mal sur des points essentiels.

C'est notamment le cas pour «l'école artisanale romantique» que je suis censé représenter. On ne peut tout de même pas m'assimiler à un romantique en ce qui concerne l'école simplement parce que, dans mon développement du terme «standard», j'oppose production industrielle et fabrication artisanale. Schneuwly fait ressortir de mon intervention l'image d'un pédagogue charismatique «qui enseigne dans un lieu protégé, loin de toute pression sociale, de concurrence et de toute sélection, selon les besoins des enfants, suivant leur développement spontané, inventant outils, méthodes et techniques». Est-ce le résultat d'une lecture attentive de mon texte? Je ne le pense pas. Il y a un certain comique à ce que Schneuwly m'assimile à Ferrière. Pour justifier une telle caricature, mes remarques concernant l'école en tant qu' «unité de vie» ne suffisent pas, puisque je ne 
cite qu'un certain nombre de points à partir d'une liste de Fend, des points qui renvoient à l'inutilité de l'école sans que je dise pour autant que l'école devrait s'y résumer. Nulle part je n'exclus que l'école doive aussi être un lieu d'apprentissage et de performance. Puisque je critique la tradition initiée par Thorndike, Schneuwly postule simplement que je me reconnais dans un autre point de vue qu'il identifie comme étant celui de Dewey. Cette distinction est presque aussi subtile que si l'on prétendait que, dans la zone linguistique germanophone, un mouvement pédagogique «Reformpädagogik» ${ }^{1}$ existait parallèlement à la pédagogie.

De me placer dans le camp des «poppériens» met en évidence à quel point la lecture de mon texte par Schneuwly est peu stimulante. Je cite Popper exclusivement afin de soutenir l'argument selon lequel la science n'est fondée ni sur des faits purs ni sur de pures idées. On peut facilement le démontrer en citant d'autres auteurs, comme le fait par exemple Jean-Luc Patry qui renvoie à Dewey et le principe de "warranted assertibility». La référence à Luhmann (1992) serait une autre possibilité: «Weder kann man von sich selbst evidenzierenden Wahrnehmungen, noch von intuitiv sicheren Axiomen ausgehen» (p. 462). Popper n'approuverait justement pas l'idée selon laquelle la science se fonde sur une argumentation discursive, puisqu'il a conservé jusqu'à la fin son principe des «Mondes 1,2 et 3 » et seulement reconnu la logique déductive bivalente comme fondement de la démonstration scientifique. Ainsi, l'argument indiscutable du relativisme et le spectre de la tour d'ivoire ne sont pas de trop aux yeux de Schneuwly pour ridiculiser ma position. Dis-je quelque part que les mesures qui sont appliquées dans le cadre de HarmoS sont sans ancrage dans la réalité? La question n'est pas de savoir si les connaissances scientifiques ont un ancrage dans la réalité ou non, mais si elles sont vraies ou fausses.

Par manque de place, je ne peux entrer en matière sur d'autres maladresses de la réponse de Schneuwly. Je mentionne cependant une dernière chose: le propre penchant pour le romantisme de Schneuwly. De voir partout des contradictions objectives («contradictions inhérentes») et de styliser l'opposition comme moteur du changement («moteur du développement») me rappelle avec force l'époque de mes études, quand de telles «grandes histoires» (Lyotard) étaient encore crédibles.

(5) À l'opposé de la critique musclée de Schneuwly, j’apprécie beaucoup la contribution de Christian Maroy et de Vincent Dupriez. Même s'ils perçoivent également dans mon texte des fantaisies régressives - ma critique se baserait «sur une conception "humaniste» et "artisanale» de l'éducation, de ses visées et modes de fonctionnement»-, ils interprètent correctement l'essentiel de mon point de vue. Comme Reichenbach, ils complètent mes remarques descriptives par une esquisse théorique qui peut expliquer pourquoi le système éducatif est tombé sous l'influence des manières de penser de l'économie. L'analyse sociologique dans laquelle ils inscrivent ma critique postule la transformation d'une organisation bureaucratique de l'école en une organisation post-bureaucratique, laquelle 
va de pair avec une perte de légitimité politique. L'affaiblissement du caractère institutionnel de l'école entraîne une orientation simpliste des directives générales selon les critères de la rationalité utilitaire. Ce qui compte n'est plus que "ce qui marche». L'école est dépassée par une culture de la performance (voir Radtke, 2002) qui semble pouvoir se passer d'un discours sur les finalités de l'école. ${ }^{2}$

Maroy et Dupriez soulignent l'accroissement du pouvoir lié au modèle postbureaucratique; il s'agit d'un pouvoir qui se répercute négativement sur la professionnalisation du métier d'enseignant. Les deux collègues se réferent également à l'appareil d'experts et de technocrates qui accompagne la réforme et sa mise en œuvre. On peut tenir compte de ces propos pour la Suisse. Malgré l'implémentation du NPM (New Public Mangement), les effectifs des administrations de l'éducation sont gonflés et dévoilent les véritables agents de l'harmonisation du système scolaire suisse. Pour la discipline des sciences de l'éducation, ce n'est pas sans ambiguïté. En effet, un appareil administratif gonflé crée également des emplois pour les diplômées et diplômés des filières en sciences de l'éducation. Inévitablement, les nouveaux collègues qui viennent d'être formés se retrouvent devant un dilemme: doivent-ils désormais se consacrer à la science (la vérité) ou à la politique (le pouvoir)?

(6) Gianni Ghisla perçoit ma critique de l' «économisation» du système de la formation comme trop vive. Mais j'ai l'impression que nos positions ne sont pas tellement différentes. Ce que Ghisla explique à propos des standards de formation correspond à mon propre point de vue. Je peux également facilement être d'accord avec sa discussion du terme de compétence. Cependant, jusqu'à la fin, son positionnement par rapport - selon ses propres termes - à la "finalisation économique de la formation, de la recherche et de l'innovation" n'est pas clair pour moi. Cela commence déjà avec les citations de Gary Becker. Est-ce qu'il les approuve? Ou est-il tout simplement fasciné par celles-ci? Celui qui explique tout par l'économie peut aussi bien tout expliquer par les idées, les apparences ou les contradictions. Mais la compréhension ne devient possible que lorsqu'il existe des différences et des divergences. L'économie doit donc aussi avoir ses limites, sinon elle n'expliquerait tout simplement rien. La confrontation avec l'économie que réclame Ghisla devrait être exactement cela: une tentative de cerner les limites de la pensée économique (aussi) à propos de la formation.

Peut-être que nos positions à ce sujet ne sont pas vraiment différentes, car plus loin Ghisla appelle à la «capacité de la politique de la formation à ne pas laisser la formation se dégrader en devenant une marchandise». Mais immédiatement après, on peut lire que les appels adressés à la politique de l'éducation «contre l'instrumentalisation» (des sciences de l'éducation) n'apporteront «vraisemblablement... pas grand-chose». Sommes-nous, ou non, livrés à l'économie et à ses moyens financiers? Puisque Ghisla me reproche de présenter les sciences de l'éducation comme une "victime d'une politique sans égards», c'est qu'il semble penser qu'il y a quelque chose à faire. Les reproches que Ghisla adresse aux sciences de l'éducation qui devraient d'abord balayer devant leur porte, contrecarrent 
sa critique. Tout n'est peut-être pas au mieux dans les sciences de l'éducation et il serait judicieux que celles-ci reconsidèrent de manière radicale ce qu'elles publient. Mais il me semble que c'est là un sujet autre que celui que je traite dans mon article. Ce serait une terrible erreur de pointer les faiblesses des sciences de l'éducation pour détourner l'attention des attaques auxquelles celles-ci sont exposées par une politique inspirée par des motifs économiques.

(7) J'ai été un peu fâché par la critique d'Esther Berner. La collègue m'attribue des choses que je ne défends aucunement et qui ne peuvent pas être tirées de mon article. Où dis-je que les compétences n'auraient «rien» à faire avec la connaissance? Où est-ce que j'exige une «nouvelle théorie de la formation qui... sépare théorie et empirie»? Où est-ce que je réclame une nouvelle vérification de la structure de la réalité pédagogique? D’où peut-on déduire que je serais un adversaire des standards de la formation, un adversaire qui s'affirme même comme tel? Pourquoi serait-ce contradictoire de critiquer la standardisation qui va de pair avec la normalisation tout en critiquant le caractère formel des niveaux de compétence?

En outre, je tiens à signaler à Berner que le fait de mettre en opposition la production industrielle et la fabrication artisanale se situe dans le contexte de l'explication du terme de standard. Que je préférerais «une compréhension des processus de l'éducation et de l'enseignement comparable à celle de la production artisanale» est une affirmation gratuite. Il en va de même pour l'affirmation selon laquelle j'exigerais la prise en compte des conditions locales. Il est vrai que je suis effectivement d'avis que l'école et l'enseignement ont toujours lieu dans des conditions qui ne permettent pas un traitement égal et une évaluation égale tant des enseignants que des élèves. Vouloir ignorer les conditions locales et situationnelles des actions pédagogiques, en plus avec l'argument de l'équité, me semble être complètement déplacé. ${ }^{3}$ Le caractère toujours contextualisé (Situativität) de l'école et de l'enseignement a en outre des conséquences pour les méthodes de recherche. Outre un nombre incalculable d'interactions, c'est avant tout la contextualisation des processus pédagogiques qui fait des sciences de l'éducation une des disciplines les plus exigeantes (voir Berlin, 2002).

De plus, Berner se plaint de ce que je ne mentionnerais pas le fait que les standards de l'éducation en Suisse soient des «standards minimaux». Même si cela était vrai - en fait, ce n'est pas vrai, car je me réfere au tout début de la première partie de mon article aux «standards minimaux ou de base» sur lesquels se fonde le projet de la CDIP - il serait évident que des standards minimaux ne peuvent pas être définis en soi. Comme Ghisla l'expose, des standards se situent dans un "continuum de qualité». Or, ce sont des points limites (cutscores) qui supposent une échelle sur laquelle ils sont inscrits selon des critères de répartition (voir Mehrens \& Cizek, 2001, p. 477) - soit en tant que standards minimaux, soit comme standards maximaux ou alors quelque part entre les deux (par exemple en tant que standards par défaut).

De même la critique disant que je supprime la «boucle de rétroaction», dans mon exposé relatif au modèle de pilotage de la politique de l'éducation, n'est pas 
compréhensible. Celui qui lit les textes concernant le monitorage de l'éducation peut difficilement ignorer comment la politique et la science devraient être liées. C'est même le point principal de ma critique! Comment un modèle cybernétique de rétroaction négative, qui apporte de précieux services pour la régulation des systèmes de réfrigération, peut-il servir à l'analyse d'un tissu aussi complexe qu'un système éducatif? Ce modèle est pour le moins trop simple pour répondre à ce qu'on exige de lui.

Je renonce finalement à dissiper les autres malentendus de la contribution de Berner. Celui qui s'engage à critiquer la "qualité d'argumentation» d'un texte et qui accuse son auteur de plusieurs "erreurs», devrait porter plus d'attention à la qualité de son propre exposé que ne l'a fait Berner.

(8) Le texte de Marcel Crahay s'inscrit dans le petit groupe de contributions qui élargissent mon analyse descriptive avec des approches explicatives. Je suis tout à fait d'accord avec Crahay lorsqu'il dit qu'une critique de HarmoS n'est pas suffisante in abstracto et qu'elle doit être incorporée dans un contexte social et historique. Comme Reichenbach ou Maroy et Dupriez, Crahay fournit ce complément de manière brillante, de sorte qu'une faiblesse apparente de mon texte est maintenant corrigée.

Crahay conteste que les réformes en cours soient sans théorie, comme je le prétends dans mon article. Ces théories seraient disponibles et proviendraient de l'économie. Sur ce point je suis toutefois d'accord. La critique de la provenance des approches de la réforme est même un élément essentiel de mon argumentation. Apparemment, je me suis mal exprimé. Ce que je pense, ce n'est pas qu'il n'y a pas de concepts théoriques, mais qu'il s'agit de théories «exogènes» dont la pertinence pour l'analyse des situations pédagogiques n'est pas prouvée. La simple transposition d'une théorie économique (ou de toute autre théorie) dans le domaine de la formation est insuffisante pour être considérée comme la base scientifique d'une réforme aussi vaste. Mon argument n'est pas que la formation et l'éducation ne peuvent pas être jugées selon des critères économiques - cela peut et doit même être fait - mais il repose sur le fait que les modèles de la pensée économique sont appliqués au système éducatif sans que la validité de telles applications ait été vérifiée de manière empirique.

(9) Je perçois l'avis de Jean-Luc Patry comme très nuancé. Bien que le style polémique de mon texte ne semble pas lui convenir, son intervention est très utile. Il met en lumière un point qui - à l'exception des remarques de Bain concernant la validité diagnostique - resterait largement absent du débat: les problèmes méthodologiques et les problèmes théoriques de mesure de HarmoS et des projets de réformes de même nature. Patry permet de comprendre pourquoi il n'est pas facile de développer des standards de formation pour toutes les matières scolaires et encore moins pour les objectifs éducatifs de l'école. Il étaye ainsi un argument essentiel de mon texte, à savoir que les standards de la formation et le monitorage de la formation mettent l'école sous une lumière qui cache beaucoup de ce qui ne peut pourtant pas en être écarté. 
Ce que déclare Patry concernant les processus input et output est approprié. En effet, les programmes scolaires (qui sont des inputs) décrivent les résultats des processus de formation. D'un point de vue linguistique, ils sont souvent formulés à l'indicatif («l'élève maîtrise les bases de calcul» ou "l'élève récite librement un poème court»). Ils anticipent ainsi ce qui doit prioritairement être atteint. Inversement, le pilotage output n'est pas un pilotage de l'output, mais continue à rester un pilotage de l'input qu'orientent les informations de l'output. Si Patry estime que la séparation entre input et output ne peut pas «être tout à fait réalisée» dans l'enseignement, il apporte de l'eau à mon moulin, car je doute justement que les simples modèles input-output soient appropriés à l'analyse des processus pédagogiques. En outre, Patry fait référence à un modèle éthique que je partage également et qui fait partie depuis Schleiermacher (1826/2000) des bases de la pensée pédagogique: personne n'a le droit de sacrifier le présent d'un enfant à son avenir. C'est pour cela aussi que l'inutilité fait impérativement partie de l'idée de l'école.

Je ne suis pas d'accord avec les louanges de Patry sur le modèle TOTE, non pas parce qu'il s'agit d'un «classique», mais surtout parce qu'il n'est pas applicable à la situation que décrit mon texte. Le modèle TOTE est un modèle psychologique dont l'unité de référence est claire: l'individu qui agit. L'enseignement constitue une autre entité, à savoir une entité sociale composée de plusieurs individus. Comme le modèle cybernétique du réfrigérateur, le modèle TOTE ne suffit pas pour comprendre la complexité d'une situation de l'enseignement.

On peut suivre la logique du "plan, do, monitor and review» (voir Gleeson $\&$ Gunter 2001, p. 145) quand les conditions sont simples, mais pas quand elles sont complexes. À cet égard, le modèle TOTE est un bon exemple de la suggestibilité des processus schématiques linéaires qui, pris isolément, semblent certes plausibles mais ne rendent pas justice aux sciences de l'éducation.

(10) Maurice Tardif m'accuse de diaboliser le projet HarmoS, un vocabulaire un peu vif qui n'est que légèrement atténué quand il constate que j'y vais «à la hache». Tardif pointe lui aussi la pensée dichotomique («système bipolaire») dans mon article; cependant, ce ne sont pas deux conceptions de la pédagogie qu'il discerne, mais un dualisme entre sciences de l'éducation et économie. Comme Crahay, Tardif rappelle les relations étroites entre l'industrialisation de la société et de la création des écoles au $19^{\mathrm{e}}$ siècle. Je suis le dernier à mettre en doute qu'existent, entre formation et économie, des liens étroits. Toutefois, je tiens aussi beaucoup à une vision systémique de leurs rapports. Dans une société fonctionnellement différenciée, les éléments du système social opèrent selon une logique propre, ce qui les rend relativement autonomes (voir Luhmann, 1997). Aucun système ne peut, par son propre code, se raccorder au code d'un autre système, ce qui définit des limites à l'économie et à la politique, non seulement par rapport à la science, mais aussi par rapport à la formation.

À cet égard, j'ai un peu de mal lorsque Tardif décrit l'éducation et l'école tels quels comme étant des réalités économiques («des réalités profondément écono- 
miques»). Certainement, le système éducatif a un coût, et c'est sans doute une des raisons majeures des réformes scolaires actuelles, ce qui est admis par différents acteurs. Cette compréhension ne doit toutefois pas conduire à ce que le système de formation soit soumis aux catégories de la pensée économique. Je ne pense pas que les écoles soient des vierges innocentes, dont les appétits charnels ne seraient réveillés que par l'économie (comme le suggère la métaphore de Tardif). Mais les universités aussi coûtent cher, et pourtant, aucune des contributions n'a défendu l'idée selon laquelle la science devrait se plier au code de l'économie. La liberté de la recherche est la condition essentielle pour que la vérité puisse se faire entendre. Que l'éducation soit soumise à des buts économiques, c'est justement ce qui ne doit pas se produire. Cela n'a rien à voir avec l'exigence d'une «science pure», comme Tardif semble l'insinuer ("vision épurée et noble»), mais avec le simple fait que la vérité - en tant que code de la science (voir Luhmann, 1992) - est liée à des conditions que ne réunissent ni le politique ni l'économique.

Comme Tardif l'expose avec raison, les «sciences de l'éducation» sont en étroite liaison avec les intérêts de l'État. Cela ne vaut pas seulement pour l'Amérique du Nord, mais aussi pour l'Europe. À cet égard, je ne prétends pas non plus que nous avons ici affaire à un phénomène nouveau. Mais je pars du principe que c'est précisément là un problème de la légitimité des sciences de l'éducation en tant que discipline académique. Contrairement à Tardif qui ne voit apparemment pas de lien, je pense que les faiblesses constatées de la recherche en sciences de l'éducation s'expliquent (aussi) par le fait que la discipline est exposée à des exigences qui ne sont pas de nature scientifique. Tardif détourne mon raisonnement en estimant que les raisons de l'incompétence épistémique des sciences de l'éducation résideraient en elles-mêmes, alors que je pense qu'elles sont conditionnées en grande partie par l'extérieur. Sur ce point, je me distingue moi aussi de tous ces collègues qui essaient de faire de la nécessité vertu, en assignant aux sciences de l'éducation, en tant que science, un caractère particulier: une science qui agit, une science pratique, une science de la pratique pour la pratique, une science des métiers pour les enseignants, etc. Je ne me trouve manifestement pas sur cette voie. Mais il me paraît exagéré de parler là d'une «illusion de jeune diplômé académique» (Criblez).

(11) Les explications de Lucien Criblez font également partie du groupe des contributions qui veulent donner un peu plus de profondeur à mon argumentation tout en cherchant à expliquer ce que je critique. Il me semble un peu étrange que Criblez parle d'une «autre lecture» puisqu'en même temps il me reproche d'être a-historique. À mon avis, Criblez ne propose pas une autre lecture; il renforce mon intervention en s'appuyant sur du matériau historique. À cet égard, je peux bien suivre son argumentation focalisée sur la spécificité de la situation politique en Suisse. Je n'ai pas affirmé que la politique de l'éducation veuille accessoirement asservir les sciences de l'éducation. Toutefois, je suis stupéfait de la naïveté qui traverse l'exposé de Criblez. Suffit-il à une analyse historique de se baser 
sur des documents officiels pour reconstruire la compréhension propre aux acteurs? Contrairement à Reichenbach, Maroy et Dupriez ou encore Crahay, Criblez n'essaie pas de prolonger mon analyse par de la théorie; il reste dans le narratif, ce qui donne à ses interventions un caractère quelque peu affirmatif.

Pour donner un exemple, il me semble discutable de considérer que les interventions de l'État ont toujours un impact, qu'il soit celui qui est attendu ou inattendu. Je suis mal à l'aise par rapport à PISA ou d'autres études sur la performance scolaire lorsque j'entends que, même si elles ne servent à rien, elles ont au moins le mérite de provoquer le débat. Cet avis, empreint de cynisme, donne raison à Reichenbach qui voit dans les réformes scolaires une sorte de coureur solitaire qui se remet toujours en mouvement car une "politique rationnelle» n'est absolument pas possible!

À cet égard, le fait que Criblez n'ait pas discuté le terme de pilotage me dérange. Quand les hommes politiques emploient un mot, on peut admettre qu'il s'agisse souvent d'une façon de parler. Mais dans la science, il devrait en être autrement. Jusqu'à quel point un "pilotage» est-il possible dans un domaine politique traversé de contradictions (Schneuwly), qui doit répondre à de multiples fonctions (Reichenbach), dont les mécanismes de fonctionnement ne sont au mieux que partiellement connus, et qui se trouve être pris dans «un conflit irréductible avec la science»(Criblez)? Même si le pilotage devait commencer seulement aux niveaux macro et méso, la question du comment et du quoi piloter reste ouverte. Criblez méconnaît également que je ne prétends pas que les réformes scolaires actuelles devraient viser le pilotage au niveau de l'enseignement. De mon point de vue, la mentalité technologique qui se répand dans la politique et la gestion de l'éducation pourrait se diffuser dans le grand public et nuire à l'image de l'école et de la profession enseignante ${ }^{4}$.

Criblez remet en question le fait de penser en termes de pilotage lorsqu'il interprète le rapport entre la science et la politique comme systémique. Mais comment peut-il réunir sous un même toit le désir d'appliquer le pilotage et le fait de se rendre compte que le pilotage n'est pas possible? Et que signifie le discours à propos du "pilotage des politiques de l'éducation" et des «savoirs à propos du pilotage» que le politique exige de la recherche en éducation - une exigence justifiée selon Criblez? Comme chez Tardif, le raisonnement de Criblez débouche sur un haussement d'épaule quiétiste: ma "préoccupation pour une autonomie des sciences de l'éducation» serait "honnête» certes, mais étant donné les conditions actuelles, nous les acceptons - avec la sérénité recommandée par Ghisla.

Criblez minimise ma critique quand il se réfere à la légitimité d'une politique qui fonde ses décisions scientifiquement. Je n'ai rien contre cela! Ma critique ne consiste pas à défendre que la connaissance scientifique puisse alimenter les décisions politiques, mais bien l'asservissement de la science par une politique qui se prétend rationnelle. L'appel aux sciences de l'éducation de renforcer «leur propre discipline» contribue peu à contrecarrer cette idée. Comme dans le cas de la 
profession enseignante, on ne peut pas être autonome si l'autonomie nécessaire n'est pas accordée. À cet égard, je ne soutiens pas l'«autonomie totale de la science». Le reproche est injuste et a pour seule conséquence l'absolution accordée au statu quo. J'ai eu l'impression que Criblez créait un écran de fumée pour que les contours de ma critique s'y perdent et que l'on revienne au plus vite à l'ordre du jour. C'est peut-être dans son intérêt; ce n'est pas dans le mien.

Encore un mot sur la «pensée nationale» que non seulement Criblez, mais aussi Schneuwly et Patry rejettent comme critique des standards de l'éducation. Ce que j'en pense ne relève toutefois pas du classement des pays ou des cantons tel que présenté par PISA. À cet égard, la référence faite à PISA dans mon texte est trompeur. Ce que je veux dire est que les réformes de l'école s'arrêtent précisément aux frontières des nations. Quand chaque pays développe ses propres «standards nationaux d'éducation", la nation se positionne bel et bien à l'avantplan, alors que dans d'autres domaines politiques, les frontières de l'État perdent de l'importance ou sont dissoutes (voir Marginson, 1999). Une politique économique motivée utilise l'éducation pour accroître la compétitivité de l'économie locale à l'échelon des marchés mondiaux. N'est-ce pas là une pensée nationale?

Un dernier point concerne la question de la légitimité démocratique d'une politique qui se réfere à des «connaissances validées» et à des "causalités prouvées». Criblez parle brièvement du problème pour le laisser tomber peu après; il est repris dans d'autres contributions, mais seulement accessoirement (par exemple chez Schneuwly par rapport au contrôle démocratique des décisions de la CDIP). Les «connaissances acquises» (Criblez utilise cette expression indicible) ne sont de loin pas des décisions démocratiques, parce que ce qui est «acquis» et "prouvé» n’a pas besoin d'être approuvé par une majorité. Dans ce sens, une politique basée sur des preuves échappe à la légitimation publique. Même si dans les nouveaux articles de la Constitution, on parle d'un «espace de fonction suisse» (art. 61a) et d'une "harmonisation de l'instruction publique» (art. 61), il n'est écrit nulle part que ce doit être au détriment du domaine politique et en pesant lourdement sur le processus démocratique. Rien que la tentative d'instrumentaliser les sciences de l'éducation dans le cadre d'une evidencebased policy ne peut pas être accepté dans un pays tel que la Suisse avec sa tradition démocratique!

\section{Notes}

1 Rien qu'en se référant aux États-Unis, cette dichotomie est fausse, puisqu’à côté de Thorndike et Dewey, il faudrait aussi prendre en compte Judd (voir Hilgard 1996; Langemann 2000).

2 Gianni Ghisla aussi mentionne et illustre par une anecdote la marginalisation de la discussion des standards et des objectifs de l'école sous le terme de «Kulturbereinigung».

3 Pour une description convaincante de la contextualité de l'action pédagogique (Situativität pädagogischen Handelns), voir Patry (1991, 2000).

4 Peut-être devrait-on éliminer le caractère métaphorique du concept de "pilotage» et parler à la place de «gouvernance» (voir Kussau \& Brüsemeister, 2007). 


\section{Références bibliographiques}

Berliner, D.C. (2002). Educational research: The hardest science of all. Educational Researcher, 31 (8), 18-20.

CDIP [Conférence suisse des directeurs cantonaux de l'instruction publique]. (2006). Accord intercantonal sur l'harmonisation de la scolarité obligatoire - Concordat HarmoS. Berne: CDIP.

Gleeson, D. \& Gunter, H. (2001). The performing school and the modernisation of teachers. In D. Gleeson \& C. Husbands (Éd.), The performing school: Managing teaching and learning in a performance culture (pp.139-158). London: RoutledgeFalmer.

Hilgard, E. R. (1996). History of educational psychology. In D. C. Berliner \& R. C. Calfee (Éd.), Handbook of Educational Psychology (pp. 990-1004). New York: Macmillan.

Kussau, J. \& Brüsemeister, Th. (2007). Governance, Schule und Politik: Zwischen Antagonismus und Kooperation. Wiesbaden: Verlag für Sozialwissenschaften.

Lagemann, E.C. (2000). An elusive science: The troubling history of education research. Chicago: University of Chicago Press.

Luhmann, N. (1992). Die Wissenschaft der Gesellschaft. Frankfurt a.M.: Suhrkamp.

Luhmann, N. (1997). Die Gesellschaft der Gesellschaft. 2 Bde. Frankfurt a.M.: Suhrkamp.

Marginson, S. (1999). After globalization: Emerging politics of education. Journal of Education Policy, 14, 19-31.

Mehrens, W. A. \& Cizek, G. J. (2001). Standard setting and the public good: Benefits accrued and anticipated. In G. J. Cizek (Éd.), Setting Performance Standards. Concepts, Methods, and Perspectives (pp. 477-485). Mahwah, N.J.: Lawrence Erlbaum.

Patry, J.-L. (1991). Transsituationale Konsistenz des Verhaltens und Handelns in der Erziehung. Bern: Peter Lang.

Patry, J.-L. (2000). Kaktus und Salat: Zur Situationsspezifität in der Erziehung. In J.-L. Patry \& F. Riffert (Éd.), Situationsspezifität in pädagogischen Handlungsfeldern (pp. 13-52). Innsbruck: Studienverlag.

Radtke, F.-O. (2002). Die Erziehungswissenschaft der OECD: Aussichten auf die neue Performanmz-Kultur. In D. Nittel \& W. Seitter (Éd.), Die Bildung des Erwachsenen: Erziehungs- und sozialwissenschaftliche Zugänge (pp. 277-304). Bielefeld: Bertelsmann.

Schleiermacher, F. (1826/2000). Texte zur Pädagogik: Kommentierte Studienausgabe, Bd. 2. Frankfurt a.M.: Suhrkamp. 